GABRIEL AMENGUAL

Universidad de las Islas Baleares

g.amengual@uib.es

\title{
Reconocimiento y solidaridad
}

\section{Recognition and solidarity}

RESUMEN: Hegel nos ha proporcionado diversas exposiciones de su teoría del reconocimiento, el cual es en sí mismo un proceso que comprende diversas fases. También ha sido objeto de múltiples interpretaciones y recepciones y todo ello contribuye a una diversidad de concepciones de tal teoría, según se acentúe una exposición u otra y una(s) fase(s) u otra(s). De hecho contamos con interpretaciones dispares según sea el aspecto que es considerado como central, sea la lucha entre el señor y el siervo, el concepto o el movimiento del reconocimiento como tal, la fundamentación de la ética y las instituciones de la eticidad, etc. Aquí no se pretende elaborar una
ABSTRACT: Hegel has provided us with different assertions about his theory of recognition, which is in itself a process encompassing various phases. His theory has been also subject to many interpretations and receptions, all of them contributing to a variaety of views about that theory, depending on the statements and or phases which were emphasized. In fact, these so many interpretations are diverse, according to the central aspect considered, be it either the lordship and bondage struggle, the notion or the movement of recognition as such, the grounding of ethics and institutions of ethical life, etc. Here it is not our aim to 
nueva interpretación, sino destacar los elementos que puedan sugerir algo acerca de la solidaridad. Ciertamente que como tal la teoría del reconocimiento no es una teoría de la solidaridad $y$, sin embargo, no resulta difícil descubrir puntos de contacto y afinidades.

PALABRAS CLAVE: AMOR; ETICIDAD; INTERSUBJETIVIDAD; CONCIENCIA SOCIAL work out a new interpretation, but just to stress the elements that can suggest a contribution to solidarity. Certainly, a theory of recognition is not a theory of solidarity, but in Hegel it is not difficult to discover points of convergence and affinities.

KEY WORDS: LOVE; ETHICAL LIFE; INTERSUBJETIVITY; SOCIAL CONSCIOUNESS

$\mathrm{L}$

A TEORÍA DEL RECONOCIMIENTo cuenta con diversas exposiciones de la mano de Hegel mismo; el reconocimiento en sí mismo es un proceso que comprende diversas fases y también ha sido objeto de múltiples interpretaciones y recepciones. Todo ello contribuye a una diversidad de concepciones de tal teoría, según se acentúe una exposición u otra y una(s) fase(s) u otra(s). De hecho contamos con interpretaciones dispares según sea el aspecto que es considerado como central, sea la lucha entre el señor y el siervo, la formación del siervo y la anulación del señor, el concepto o el movimiento del reconocimiento como tal, la teoría de la intersubjetividad, la fundamentación de la ética y las instituciones de la eticidad, la fundamentación del derecho y del Estado como sustitución de la teoría del contrato, la relación entre individuos por la que éstos se forman y a la vez se crea la voluntad general, etc. ${ }^{1}$

Aquí no se pretende elaborar una nueva interpretación, sino destacar los elementos que puedan sugerir algo acerca de la solidaridad. Ciertamente que como tal la teoría del reconocimiento no es una teoría de la solidaridad y, sin embargo, no resulta difícil descubrir puntos de contacto y afinidades. Entre las cuales destaca que quiere ser la respuesta a la misma pregunta (o parte de ella) a la que quiere también dar respuesta la solidaridad, así como presenta una potente teoría de la intersubjetividad, de modo que cada autoconciencia es formada por su relación con otra autoconciencia. De modo general, de entrada, hay que recordar que la teoría del reconocimiento pretende insertar en el paradigma iusnaturalista moderno, el del individualismo, la sociabilidad de la que partía

[1] Para una visión esquemática del concepto cf. Amengual (1990). Para una exposición sucinta, pero siguiendo toda la evolución del pensamiento de Hegel cf. MAZA (2009). 
la política clásica, de cuño aristotélico. Consideremos, pues, en primer lugar, el problema al que pretende responder (1). Después se expondrá la teoría del reconocimiento desde la perspectiva de nuestra búsqueda (2), para finalmente recoger aquellos elementos de la teoría del reconocimiento que pueden tener su lugar y su función en el concepto de solidaridad (3).

\section{EL PROBLEMA AL QUE QUIERE RESPONDER}

La teoría del reconocimiento se desarrolló en el idealismo alemán para evitar una concepción individualista, que reduce el Estado a una asociación de intereses, como también para evitar una concepción colectivista, que supedita el individuo a las exigencias de grupos, sean asociaciones de familias, comunidades religiosas, corporaciones o Estados (Siep 2009, 15 s.). Quiere dar una respuesta al hecho que la pérdida de la eticidad de la polis griega es irrecuperable, como constata Hegel en Jena, a pesar de ser considerada un ideal. Pero tampoco satisface el individualismo moderno y las respuestas contractualistas que se la han dado. El individualismo es un hecho evidente y que se impone en el modo de concebir y manejar las relaciones sociales. Y, por otra parte, la solución sobre la base del contrato, especialmente en su versión hobbesiana, siempre llega tarde y es extrínseca al ser del individuo y de la comunidad: una vez ya formado el individuo, sea de un modo u otro, se decide a unirse con los demás sin más motivo que sus necesidades, para mejor satisfacer necesidades de todo tipo. El contrato aparece siempre más bien como un elemento administrativo, pero sin carácter ético. «Por medio de este principio [del reconocimiento] Hegel quiere pensar conjuntamente el 'moderno' concepto de libertad con los rasgos fundamentales de la eticidad de la polis» (Siep 1979, 18), lo cual por otra parte, es una característica de toda la filosofía práctica hegeliana (Amengual 2000).

En los últimos años de Jena el modelo clásico griego de la política, de corte radicalmente comunitarista, entra en crisis y por primera vez en las lecciones de 1805-06 se declara la superioridad del principio moderno de la libertad individual, con lo cual se viene a declarar la eticidad clásica como un mundo definitivamente perdido. Así Hegel afirma: «Tal es el principio superior de la época moderna, que los antiguos, que Platón no conociera. En la edad antigua la bella vida pública era la costumbre de todos; la belleza constituía la unidad inmediata de lo general y lo individual [...]. Pero no se daba un saber de la individualidad absoluto para sí mismo, este absoluto ser-en-sí. La república platónica, como el estado lacedemonio, es esta desaparición de la individualidad que se sabe a sí misma. Con el nuevo principio, en cambio, se pierde la libertad externa y real de los individuos en su existencia inmediata, pero se gana la interna -su libertad de pensamiento-, el Espíritu queda purificado de la existencia inmediata y ha entrado en su puro elemento del saber, indiferente 
con respecto a la singularidad existente. Aquí comienza a ser saber, o se trata de su existencia formal como saber de sí mismo.» (GW VIII, 263 s. / 215 s.) ${ }^{2}$

La teoría del reconocimiento surge, pues, como un intento de dar respuesta a una búsqueda epocal; en el fondo se está buscando una nueva forma de vida, que conjugue satisfactoriamente la afirmación del individuo y su libertad, «el principio superior de la época moderna», y a la vez su dimensión social, es decir, su pertenencia intrínseca a la comunidad o a diferentes instancias sociales y políticas. El reconocimiento viene, pues, a sustituir el contrato, ${ }^{3}$ por su mayor fuerza de cohesión social y por su intervención en la misma formación del individuo, el cual se forma precisamente mediante el proceso de reconocimiento, así como mediante el proceso de formación de la misma comunidad en la que el individuo se encuentra inserido. Según la teoría del reconocimiento no se trata de que el individuo se insiera en la comunidad, sino que ya se ha formado en ella, formando parte de ella, de modo que ésta le constituye: los otros, la comunidad la lleva dentro; su formación ha sido a la vez y por el mismo movimiento un proceso de socialización y un proceso de individualización.

Por tanto, «con el principio 'reconocimiento' Hegel no ha querido universalizar la estructura de un tipo particular de acción o de las relaciones sociales, sino que ha intentado indicar la estructura de un proceso de formación de la conciencia singular y de la común, proceso que determina las diversas formas de interacción y relaciones sociales de lenguaje, trabajo, amor, contrato, intercambio, derecho, etc., según su respectiva índole» (Siep 1979, 17 s.). No se trata, pues, ni de una acción o tipo de acción, ni tampoco de una relación social, como tampoco se trata de un principio formal o procedimental que haya que cumplir, sino que se cumple mientras tiene lugar la formación de la conciencia individual y la común o universal; y en la exposición del movimiento del reconocimiento no se hace independientemente de la exposición de las instituciones y su génesis histórica.

No resulta difícil establecer paralelismos y afinidades con las funciones que el concepto de solidaridad está jugando en nuestro presente. Tras dicho concepto en nuestro tiempo está la búsqueda de una forma de vida, que consiga conjugar o equilibrar y armonizar tendencias al menos en parte contrapuestas, como son, por una parte, la tendencia a una mayor individualización, la afirmación de la singularidad del individuo y del carácter personal con que se quiere impregnar toda su vida, incluidas sus creencias y sus pertenencias a los diversos colectivos o instituciones. Dicha tendencia, por otra parte, va acompañada de la búsqueda de cohesión social que supere exclusivismos tanto

[2] Cf. el estudio sobre un escrito anterior de Hegel y el contexto cultural del surgimiento del individualismo moderno de BOdeI (1987); y más en general D’AbBiero, VINCI (1995).

[3] La confrontación de Hegel con Hobbes en SIEP (1974). 
de raíz económica, social, racial o de género, como de raíz cultural y política; la búsqueda de fines y motivos que puedan promover y orientar una acción social cooperativa que brinde nuevas oportunidades a los que carecen de ellas. Por otra parte está toda su carga social y política en su sentido más global, que tiene que ver con la distribución de la riqueza y de la mejora de las condiciones de vida, de lucha contra la pobreza, el hambre y la enfermedad, de acogida de emigrantes y refugiados, de discapacitados y ancianos y tantos desfavorecidos por mil circunstancias.

\section{LA TEORÍA DEL RECONOCIMIENTO}

Antes de entrar en la valoración de la presencia de la dimensión de solidaridad en el reconocimiento, conviene recordar las líneas generales de la teoría del reconocimiento para poder evaluar mejor su eventual aportación al concepto de solidaridad. Según la exposición más completa de la teoría del reconocimiento en la Filosofía real (1805-06), cabe distinguir dos fases o grados en el proceso del reconocimiento: por una parte, el reconocimiento como relación recíproca entre individuos y, por otra, la relación de reconocimiento entre la voluntad singular y la universal, entre el sí mismo formado y el espíritu del pueblo (Siep 1979, 53 s.).

\section{II.1. PRIMERA FASE: RELACIÓN RECÍPROCA ENTRE INDIVIDUOS II.1.1. Según la Filosofía REAL (1805-06)}

La primera fase del proceso de reconocimiento consiste en la relación recíproca entre individuos, en la cual mediante el conocer recíproco y el obrar entre individuos surge una conciencia común, una voluntad universal. En dicho desarrollo aparecen como necesarios dos movimientos diferentes, que Hegel concibe como una relación a dos. De hecho en la Filosofía Real el «ser reconocido» de la "persona» se forma mediante dos silogismos: 1) el amor como «ser reconocido sin la oposición de la voluntad» (GW VIII 218 / 178) y 2) la lucha como «reconocimiento» de los sujetos como «sí mismos libres» (GW VIII 221 / 180).

\section{EL AMOR}

El amor del que trata Hegel es el amor de familia (Düsing 1986, 308-312). El reconocimiento en la familia tiene, en efecto, como fundamento general el amor y en concreto entendido como una determinidad específica de la voluntad. En el amor el yo se ve a sí mismo inmediatamente en el otro y al revés (el otro en el yo primero). Ambas partes tienen una relación inmediata, que Hegel posteriormente denominará eticidad natural (familia), en la que no se da ningún 
saber explícito de sí ni de la unidad de ambos. Ello se obtendrá mediante un tercero: el hijo (GW VIII 212 / 174). En la medida que el hijo ha devenido una persona consciente se da la «superación del amor» (GW VIII 213 / 174) como una relación inmediata.

La vinculación por el amor en la familia es según Hegel «estar inmediatamente reconocido» (GW VIII 238 / 194). Cuando el hijo se ha convertido en un ser independiente y autoconsciente el reconocimiento natural se convierte en espiritual (GW VIII 213 / 175). Con ello cambia el modo del reconocimiento: los individuos, que se reconocen espiritualmente unos a otros, actúan como personas libres y autoconscientes.

\section{LA LUCHA Y EL RECONOCIMIENTO JURÍDICO}

Si el amor es el perderse en la unidad del amor ¿cómo puede darse una retirada de modo que se llegue a una confrontación, a una lucha? (Siep 1979, 63-68). El paso del amor a la lucha se da por medio de la familia, la cual introduce un tercero: el hijo y el patrimonio, como objetivaciones del amor de ambos. El patrimonio objetiva sólo momentos del amor, como servicio mutuo. En el hijo el amor encuentra su objetivación propia. Dicha objetivación sienta también una distancia. El proceso generativo es tanto superación de una individualidad como configuración de una nueva. En el hijo los padres contemplan su sí mismo común objetivado y devenido ser-otro. Tanto la conciencia de los padres -en su relación con el hijo y con el patrimonio- como la del hijo se convierten en totalidad, un "ser-para-sí autoconsciente», una «individualidad plena, libre» (GW VIII 213 / 175). De este modo ya en las relaciones internas de la familia aparecen momentos de la relación de totalidades independientes, que según Hegel necesariamente deben ser de «lucha» (GW VIII 213 / 174).

De ahí surge un segundo modo de reconocer entre personas con voluntad libre y acción: el reconocimiento jurídico. Este no parte de una unidad, como el amor, sino al contrario de que cada individuo aposta por sí mismo y excluye al otro. Se presupone la voluntad libre, aunque ésta es solo formalmente libre. La relación es recognoscente (GW VIII, 215 / 176).

La relación recognoscente es, primeramente, relación entre personas libres (GW VIII, 215 / 176). En segundo lugar, el reconocimiento recae en la posesión de cosas. Este derecho abstracto precede a la institución del Estado. Ambas significaciones de reconocimiento jurídico están conectadas. De hecho una molestia o violencia física, aunque sea sobre cosas poseídas, es a la vez una vulneración incluso de la persona, de su derecho y dignidad. De esta manera surge la lucha a vida o muerte. Esta lucha termina con que cada uno conoce y sabe al otro como un libre ser-para-sí. La voluntad que se reconoce a sí y al otro como ser-para-sí es la voluntad que sabe y es universal. La particular sin- 
gularidad de la voluntad de una persona es superada; la persona misma quiere lo universal, el derecho, y por ello es espiritual. La persona, constituida por medio del reconocimiento, no desaparece en una universalidad superior como en una sustancia, por ejemplo en el espíritu del pueblo. La universalidad se ha de constituir desde la voluntad de los singulares (GW VIII 257 / 210); tengo «mi positivo sí mismo en la voluntad común» (GW VIII 255 / 209). Se reencuentra en la universalidad como independiente. Esta es la voluntad subjetiva que los antiguos no conocieron, que consiste en cuanto a su contenido en la disposición de ánimo (Gesinnung) subjetiva, interior, de obrar de acuerdo con las costumbres del pueblo.

\section{II.1.2. Según la Fenomenología}

Esta primera fase del reconocimiento se encuentra ampliamente expuesta en la Fenomenología y exclusivamente presentada como relación entre dos autoconciencias, sin concluir en forma alguna de institución o voluntad común o general, aunque sí en la universalidad del pensamiento, «conciencia pensante en general» (FDE 138 / 122). La exposición por antonomasia es la de la primera parte del Capítulo IV (IV A).

Empecemos por observar el significado de dos términos: el de conciencia y el de reconocimiento. Respecto de la conciencia, destaca la amplitud del concepto, que no integra solamente aspectos cognitivos, sino también «formas de la conciencia práctica (apetencia, temor), de las relaciones sociales (dominio y servidumbre), de la religión y de la historia.» (Siep 2000, 97 / 98). Con el término reconocimiento Hegel se refiere al verdadero concepto de las relaciones sociales. «'Reconocer' implica una constitución plural, intersubjetiva de la autoconciencia, una relación de yo y tú así como entre yo y nosotros» (Siep 2000, 99 / 99). ${ }^{4}$ El 'movimiento del reconocimiento' no está limitado a la lucha por el reconocimiento. Éste es, más bien, el telos, la meta que debe lograrse a través de todas las fases del desarrollo del espíritu y que, en todo caso, sólo se logra en el último capítulo de la Fenomenología. Por ello «Hegel ve realizado el concepto de reconocimiento en una comprensión determinada del derecho moderno, de la moralidad y de la religión» (Siep 2000, 98 s. / 98).

[4] Honneth 1994, 72 s. / 58 s. ha puesto de manifiesto desde otros puntos de vista, propios de otras ciencias humanas, como la psicología y la sociología, la amplitud del concepto de reconocimiento, de modo que pertenece esencialmente a la autoconciencia de individuos y grupos la 'aspiración' a ser reconocido por los otros. 


\section{Movimiento DE DOBLE SENTIDo}

El reconocimiento en la Fenomenología se encuentra expuesto casi exclusivamente como relación entre dos autoconciencias. La autoconciencia no se encuentra sólo con objetos exteriores ni con los que provocan su apetencia, ni siquiera con seres vivos, sino también con seres autoconscientes. Por el encuentro con otra autoconciencia la primera alcanza su propia autoconciencia, es decir, se constituye como tal.

El primer momento del movimiento del reconocer es el ser «fuera de sí», la salida de sí y la pérdida de sí mismo en el otro. Pero con ello también el otro pierde su independencia: en la medida que el sí mismo se encuentra en el otro, este otro mismo es superado, pierde su carácter de otredad, porque en él se encuentra el yo. «Para la autoconciencia hay otra autoconciencia; ésta se presenta fuera de sí. Hay en esto una doble significación: en primer lugar, la autoconciencia se ha perdido a sí misma, pues se encuentra como otra esencia; en segundo lugar, con ello ha superado a lo otro, pues no ve tampoco a lo otro como esencia, sino que se ve a sí misma en lo otro» (FDE 128 / 113). Este movimiento tiene algo que ver con la estructura del reconocimiento del amor, aunque aquí no se diga nada de la relación positiva con el otro (Siep 1979, 69).

En un segundo momento, contra la pérdida de sí mismo, se hará valer la certeza de sí mismo como esencia, es decir, la independencia. Ello acaece en una relación negativa, excluyente con el otro, que no puede ser independiente donde estoy yo, a saber, ahora también en el otro. Este es el punto de partida para la lucha, en la cual la superación del otro se convierte en autosuperación.

Lo que en la experiencia de la conciencia, que realiza el movimiento del reconocimiento, acaece como una cosa después de la otra, en el concepto del reconocer es lógicamente simultáneo, por ello Hegel lo presenta como un doble sentido. El doble sentido consiste en que lo que hace cada uno para con el otro -la negación del otro- también lo hace para consigo -negación de su ser otro o de su existencia externa. También significa que el hacer de cada uno -para consigo y para el otro- al mismo tiempo es el hacer del otro. El movimiento del reconocer se representa como el hacer de una autoconciencia, pero esta acción es «tanto su hacer como el hacer de la otra». «El movimiento es, por tanto, sencillamente el movimiento duplicado de ambas conciencias. Cada una de ellas ve a la otra hacer lo mismo que ella hace; cada una hace lo que exige de la otra y, por tanto, sólo hace lo que hace en cuanto la otra hace lo mismo» (FDE 129 / 114). «Se reconocen como reconociéndose mutuamente» (FDE 129 / 115). 


\section{LUCHA}

Pero este hacer de cada una de las autoconciencias incluye la relación excluyente de la otra para mostrar la propia independencia. El intento de mostrar como nulo el ser-otro del otro, conduce a la lucha a vida o muerte, que pone en peligro a cada uno de ver negado su propio ser-otro, su vinculación con las cosas y la existencia corporal. Un peligro que o bien conduce a la subordinación del sí mismo bajo este ser-otro -como es el caso del siervo, que de esta manera sucumbe a dicho peligro-, o bien conduce a la consciente negación del propio ser-otro (de la vida), cuando la autoconciencia es preferida a la 'vida', como es el caso del señor. Solamente cuando ambas, una a la otra, se hayan presentado como "puro ser para sí, es decir, como autoconsciencias» (FDE 130 / 115), y cuando saben que esta exposición del otro puede reflejar y reconocer su propio hacer, se llega a la conciencia de la unidad del yo y del nosotros, al concepto de espíritu (FDE 127 / 113) (Siep 1979, 70 s.). El reconocimiento se nos presenta aquí como lo que «traba las autoconciencias entre sí y constituye la unidad del espíritu. La perfecta libertad e independencia de los sujetos en el seno del espíritu se realizará en el reconocimiento mutuo» (Valls 1971, 111). La realización de esta unidad del yo y del nosotros nos adentra ya en la segunda fase del reconocimiento, en la que se da la relación entre el yo, la voluntad singular, y el nosotros, la voluntad universal.

\section{II.2. SEgunda FASE: RECONOCIMIENTO DEL YO EN EL NOSOTROS}

\section{II.2.1. Según la Filosofía ReAL}

Para la exposición de la segunda fase, la del reconocimiento del yo en el nosotros, volvemos a la Filosofía Real, al esbozo de sistema de 1805-06.

En el capítulo "Estado de reconocimiento» (Anerkanntsein, GW VIII 223-236 / 182-192) ya se nombran instituciones. Se empieza con el concepto de persona, como el ser-reconocido. La persona es la primera figura jurídica, figura o determinación de la voluntad general, que se define por ser reconocida, reconocida por sí misma como ser libre e independiente, sujeto de derechos y deberes, como fuente y fundamento del derecho, como dirá posteriormente Hegel (FD \$ 36). A la persona le siguen las figuras del trabajo, el contrato, crimen y castigo, correspondiendo en líneas generales a las del «Derecho Abstracto» de la Filosofía del Derecho.

A pesar de que el derecho contenga la oposición de las voluntades singulares, que se manifiesta en la lucha, las primeras formas con que aparece el derecho se han de considerar como una concordancia inmediata. Lo común a ellas es la concordancia de las voluntades de todos en el acto particular de la voluntad de los singulares. De esta manera el singular en su trabajo abstracto, en la medida en que el trabajo está referido a las necesidades de todos, puede 
contemplar la «universalidad de sí mismo» (GW VIII, $226 \mathrm{R}$ / 184 nota 3), que consiste precisamente en el consentimiento implícito de todos en dicho trabajo. Este consentimiento es 'puesto' en el intercambio. El intercambio no es ningún movimiento de distanciamiento consciente, sino que repite solamente un momento de la lucha -la recíproca y mediada autonegación- en el elemento de la concordancia sin oposición. Precisamente este ser mediado por la voluntad de los demás -y por cierto no de un otro, sino de todos los otros- es el ser reconocido (Anerkanntsein). El movimiento del distanciamiento (de lucha) se da en la ruptura del contrato, por la que una voluntad vuelve a sí. Por este ir en sí (Insichgehen) de la voluntad aparece una diferencia entre la voluntad singular y la universal. Cuando la voluntad se niega a participar en el rendimiento común, entonces la voluntad común obliga. En esta obligación o coerción (Zwang) se muestra el verdadero sentido del contrato. De ahí se pasa de la violación del contrato y la coerción directamente al delito y la pena como formas superiores de la lucha entre la voluntad singular y la universal (Siep 1979, 88 s.).

La estructura del reconocimiento incluye un movimiento de escindir la unidad inmediata de la voluntad singular y la universal por medio de un 'retorno' de la singularidad en sí. Ahora bien, la unidad inmediata, de la que se partió, y la escisión a la que se ha llegado no son sino como los momentos del movimiento 'dialéctico' del espíritu. La estructura del movimiento del reconocer se corresponde con la del espíritu, que se autodiferencia o se escinde y se reconcilia. «Reconocimiento es el espíritu bajo el aspecto de la formación de la autoconciencia en interacciones entre individuos así como entre individuos e instituciones» (Siep 1979, 93). Reconocida es precisamente la independencia, que incluso puede retirarse de la unidad con la voluntad de todos.

La relación entre singular y universal presenta dos aspectos. Por una parte hay un aspecto positivo, por el que el sí mismo se encuentra en el universal en la medida que sabe que la voluntad común es puesta "por mí». Lo universal tiene su vida solamente en el sí mismo de los individuos. Es el momento de la afirmación del singular en el universal mismo. Por otra parte, la positividad también puede verse por el lado opuesto, a saber, en la actividad que el universal realiza a favor del singular, como la misma protección de su existencia. Pero también se da el aspecto negativo, por el que el universal es poder sobre mi vida, de modo que el singular ha de someterse al universal (Siep 1979, 94 s.).

Dada esta ambigüedad insuperable que late en todo el proceso del reconocimiento en el nivel de la realización individual y colectiva, es decir, ética y política, este ámbito no puede ser el lugar del reconocimiento pleno, sino que finalmente el singular se sabe absolutamente libre en la religión y en la filosofía (Siep 1979, 96). 
En definitiva el reconocimiento consiste, por tanto, en una unidad de sí mismo individual y universal, positiva y negativa, inmediata y 'formada', es decir, pasada por la negación de la singularidad en el sentido de exteriorización y autorrenuncia. Su importancia se hace patente al considerar que el ser reconocido del individuo en el Estado es una relación de constitución recíproca (Siep 1979, 97).

\section{II.2.2. Según la Fenomenología}

En la Fenomenología la relación entre las autoconciencias aparece como una anticipación del concepto de espíritu (FDE 127 / 113). Dicho concepto aparece cuando la autoconciencia tiene como objeto a otra autoconciencia, porque en realidad ya se da una comunidad de autoconciencias que se reconocen. Por tanto, «la pregunta por el reconocimiento surge» no sólo «cuando una autoconciencia singular se relaciona con otra autoconciencia singular», sino «cuando en el espíritu de una universalidad intersubjetiva está implícita la relación recíproca de diversas personas singulares. - El reconocimiento presupone la existencia de intersubjetividad.» (Düsing, 313) Ello explica que "para nosotros» «esté presente ya el concepto del espíritu» (FDE 127 / 113), el cual es la unidad de individuos autoconscientes: "el yo es el nosotros y el nosotros el yo» (FDE 127 / 113). Aquí claramente se transforma el modelo o paradigma de la conciencia o subjetividad por el de la intersubjetividad, gracias a la elaboración del concepto de espíritu, que tiene la capacidad de autodiferenciarse, de salida de sí, exteriorizarse y retorno a sí, de manera que entra en relación con otro (Honneth 1994, 54 / 45 s.).

Ahora bien, ¿cómo se despliega el proceso del reconocimiento? (Düsing, 316 s.) El movimiento del reconocer es, en primer lugar, una relación entre conciencias individuales, las cuales se forman a sí mismas mediante y en esta relación a la vez que forman una relación entre ellas, en la que "para nosotros» aparece ya el concepto de espíritu. En efecto la relación entre las conciencias tiene lugar sobre un suelo común que posibilita la relación a la vez que es resultado de la relación; es «la unidad espiritual» (FDE 128 / 113);

a) «la unidad espiritual», como suelo común, posibilita la relación porque es el hecho que congrega a las conciencias singulares con las mismas pretensiones, los mismos procedimientos y fines; suelo, como si fueran las obviedades presupuestas y compartidas;

b) «la unidad espiritual», como suelo común, también es resultado de las relaciones, porque la unidad es el fruto de las relaciones, a partir de las cuales aparece ya el concepto de espíritu. 
En cambio en el reconocimiento en la Fenomenología no aparece propiamente dicha la relación vertical entre la conciencia singular y la universal, a pesar de las alusiones al concepto de espíritu.

En definitiva cabe concluir que la lucha a vida o muerte presenta un intento fracasado de reconocimiento (Düsing, 318-323). Cierto que en un primer momento el señor parece como reconocido, pero se muestra en seguida como un reconocimiento vano: «Se ha producido solamente, por tanto, un reconocimiento unilateral y desigual» (FDE 133 / 118). Por su parte el siervo se forma obteniendo así una cierta independencia, pero en todo caso no se da el reconocimiento interpersonal, y por lo que respecta a su formación, que en principio es su parte positiva, ésta no ha alcanzado sino «una habilidad capaz de ejercerse sólo sobre algo, pero no sobre la potencia universal y la esencia objetiva total» (FDE 136 / 121). De principio a fin es una relación asimétrica, en la que no se da lo que se propuso como la estructura y prueba del reconocimiento: «cada una de ellas [autoconciencias] ve a la otra hacer lo mismo que ella hace, cada una hace lo que exige a la otra» (FDE 129 / 114); «se reconocen reconociéndose» (FDE 129 / 115).

Más allá del capítulo IV, en la Fenomenología el reconocimiento del yo en el nosotros encuentra el lugar más apropiado en las figuras de conciencia y figuras de mundo, en las que ocasionalmente aparece el reconocimiento continuando el camino de la conciencia (Düsing, 318-323), a saber en la eticidad, la moralidad y la reconciliación, en definitiva en la conciencia moral (Gewissen) y el ser reconocido religioso.

\section{ELEMENTOS DE SOLIDARIDAD}

\section{III.1. A PARTIR DE LA EXPOSICIÓN HEGELIANA}

No resulta difícil percibir atisbos del concepto de solidaridad en el desarrollo del movimiento del reconocimiento. Intentemos destacar algunos.

El primer germen se encuentra ya en el mismo punto de partida, que es comprendido como «unidad espiritual» (FDE 128 / 113), como caracterización de la relación entre las conciencias, la cual se despliega sobre un suelo común, que hace posible dicha relación, porque es el hecho que congrega a las conciencias singulares con las mismas pretensiones, los mismos procedimientos y fines; suelo, como si fueran las obviedades presupuestas y compartidas y la misma necesidad de reconocer y ser reconocido. En la Filosofía Real el punto de partida es el amor familiar, que crea eticidad natural, una unidad inmediata que será objeto de una elaboración pasando por una escisión de la unidad inmediata, una superación del amor, cuando todos sus miembros sean personas independientes y libres. 
Es como si Hegel sacara consecuencias éticas del hecho antropológico fundamental de que nacemos de otros, de que el nacer es un verbo pasivo, y de que empezamos a crecer gracias al cuidado de otros, pues de lo contrario no subsistiríamos. Es un principio ético fundamental, universal y natural, que Hans Jonas formula de esta manera: «En la moral tradicional encontramos un caso [...] de una responsabilidad y un deber elementales no recíprocos, que se reconocen y practican espontáneamente: la responsabilidad y el deber para con los hijos que hemos engendrado y que perecerían sin los cuidados que a continuación precisan.» (Jonas 1994, 83) Pero es también el reconocimiento por el amor entre los esposos y entre los padres y los hijos. Este reconocimiento, como ha destacado A. Honneth, configura la estructura psíquica de los hijos y su subjetividad según el afecto de que han sido objeto (Honneth 1994, 62-68, 153-172 / 51-55, 116-132).

El amor entre los esposos, a pesar de su base natural, es un verdadero reconocimiento, una relación natural que se ha convertido en espiritual, ética. En ellos se da la reciprocidad de las consciencias que ha destacado Hegel en la Fenomenología. Es una relación personal y sin embargo no es en favor de un desfavorecido, por lo que se mueve dentro de las coordenadas de la solidaridad entendida más bien desde la esencial dimensión social de la persona y como cohesión social.

Incluso esta forma primaria de reconocimiento y solidaridad hace patente «la dependencia recíproca del reconocimiento de los derechos de los individuos y el despliegue de determinados grupos. Sin el apoyo emocional y el cuidado obvio, no concertado con contratos, en una familia o en otros grupos primarios, el individuo no puede obtener ningún sentimiento de autoestima ni ninguna estabilidad psíquica.» (Siep 2009, 16 s.)

Estas formas de reconocimiento y solidaridad, que en términos generales caen bajo la forma de reconocimiento como amor, ponen de manifiesto que «el reconocimiento presupone la existencia de intersubjetividad,» (Düsing 1986, 313) siquiera sea la esencial dimensión social de la persona, una intersubjetividad como unidad espiritual inmediata, eticidad natural, pero que se desarrolla como eticidad y como espiritual.

Al no aplicar Hegel esta forma de reconocimiento como amor a las relaciones sociales y políticas, uno no puede menos de pensar en uno de sus mayores inspiradores, Aristóteles, el cual sí habla de amistad como virtud social (EN VIII, 1, 1155a 21-29). Aristóteles recuerda el sentido social y político del amor como amistad, que no se circunscribe a las relaciones personales ni a las familiares.

Si ya en el mismo inicio de toda forma de reconocimiento se percibe la existencia de la intersubjetividad, ésta es el eje en torno al cual gira todo el proceso, el movimiento del reconocer. 
La intersubjetividad es un planteamiento básico tanto en el reconocimiento como en la solidaridad. Se la podría caracterizar como el suelo común o la estructura básica, que activa sus potencialidades a lo largo del movimiento del reconocer y de la solidaridad. - Hegel empieza su exposición de la teoría del reconocimiento en la Fenomenología trazando un esbozo de la génesis de la conciencia intersubjetiva: «La autoconciencia es en y para sí en cuanto que y porque es en sí y para sí para otra autoconciencia» (FDE 127 / 113). Lo cual significa que en la misma constitución o formación de la propia autoconciencia interviene otra autoconciencia. «No es posible un sí mismo monológico, solipsista. Seres autoconscientes son esencialmente 'para un otro' sin perder por ello su autonomía»; ello no significa estar ante un espejo, sino que también el otro depende en su identidad de otros de múltiples maneras. «Hegel habla de un 'hacer de doble sentido' en el que el hacer y el pensar de uno tiene siempre, al mismo tiempo, sus correspondencias en el hacer y pensar del otro» (Siep 2000, $101 / 101)$.

La forma más plena de intersubjetividad es la reciprocidad que se da en la acción de las dos autoconciencias. Ahora bien, la reciprocidad no surge solamente en la acción de ambas autoconciencias, sino ya en el momento original, de modo que ésta ejerce de medio en el interior del cual se forman las mismas autoconciencias. Se trata, pues, de una reciprocidad que es constitutiva de las autoconciencias, de modo que éstas llevan en su propio interior la acción de las otras; la propia identidad se forja en la relación recíproca con las otras autoconciencias. En este sentido afecta al mismo ser de la subjetividad, que no se la puede entender sin comprender a las demás con las que se ha formado, interactuando con ellas.

Partiendo de esta constatación, la solidaridad no puede ser algo ocasional y exterior, de ayuda externa, sino algo que brota del propio ser intersubjetivo de la subjetividad misma; relacionándose con los demás, se relaciona consigo. La solidaridad viene a definir la propia identidad, no es pues una necesidad que brote solamente del otro, sino del propio ser.

A partir de este ser-reconocido/solidario constitutivo de la autoconsciencia se ha de plantear el vivir de modo reconocido/solidario, es decir, el lugar y el ejercicio de la solidaridad tanto en el proceso de autorrealización personal como de realización de la comunidad/sociedad/Estado en el que se vive. La teoría del reconocimiento ofrece, pues, sobre todo una gran fundamentación de la solidaridad, poniéndola en el mismo ser de la autoconciencia, de la persona. Ella puede ser una buena fundamentación del mutualismo, en el que tantas veces se ha visto el ejercicio de la solidaridad.

Por otra parte la reciprocidad tiene también sus límites. Como ha afirmado Jonas, el mejor ejemplo de responsabilidad a favor de otros, como es la de los 
padres, es una responsabilidad que no se basa en la reciprocidad, sino que se ejerce a fondo perdido. Y el ejercicio de la responsabilidad a fondo perdido es precisamente una de las características de la solidaridad, que no encajan con el principio de la reciprocidad. Ésta es muy útil para las relaciones sociales cooperativas, cuyo principio es 'si tu ganas, yo también gano', pero no para las solidarias, para las cuales rige el principio 'yo pierdo para que tu ganes'. La reciprocidad, por otra parte, parece más bien referirse a las relaciones entre individuos, entre individuos y grupos y sobre todo entre grupos, de modo que la reciprocidad puede considerarse como el gran principio que ha regido todo el ámbito de la acción humana en la modernidad, desde la ética, la política, el derecho, hasta la economía. Al principio de reciprocidad le escapa el ámbito más propio de la acción solidaria, a saber, aquella que se hace a favor del desfavorecido por las razones que sean, el singular amenazado, marginado.

De entrada lo que parece más difícil de integrar en un concepto de solidaridad es la lucha a vida o muerte y sin embargo algo de esta dimensión se da siempre y necesariamente en toda relación solidaria. En Hegel dicha lucha emerge por la defensa de la propiedad y del honor. En el caso de la solidaridad la lucha tiene los mismos objetivos: mejor distribución de la riqueza e igualdad en dignidad para todas las personas, la cual implica una mejor capacitación para el desarrollo de las propias capacidades y posibilidades, igualdad de derechos y de oportunidades. Esta igualdad no tiene nada que ver con un retroceso a un estado de naturaleza, inmediato e indiferenciado, al contrario, es un estado logrado gracias a la lucha, es una igualdad ganada y adquirida por la lucha también a favor del reconocimiento de las diferencias y peculiaridades culturales.

\section{III.2. DIVERSIFICACIÓN DE MODOS Y GRADOS DEL RECONOCIMIENTO}

Si a partir de la propuesta de Hegel se han podido recoger estos elementos, una mirada más amplia -menos literal- al significado del reconocimiento y desde planteamientos actuales, es decir, desde el actual «grado de la 'historia de aprendizaje' de la humanidad», ha llevado a Siep a formular nuevos aspectos del reconocimiento que de hecho se pueden considerar como tantos otros aspectos de la solidaridad. De hecho su punto de partida es la «solidaridad fundamental» (Grundsolidarität) (Siep 2009, 24) que se desprende del planteamiento de reciprocidad que domina todo el desarrollo del movimiento del reconocer. Así distingue cinco grados, de menos a más, o de más elemental a más comprometido. No olvida siquiera lo que podría llamarse el grado cero, en el que se encuentran las relaciones con aquellos que uno no puede reconocer, porque uno no los soporta o los rechaza. Pero con el trato con esta clase de personas la humanidad ha aprendido que se requiere una base jurídica sobre la que poder 
actuar, partiendo de una voluntad común, que obligue a unas reglas de juego de respeto recíproco. Se trata de una solidaridad básica (Grundsolidarität).

A partir de este grado, dado por la historia de aprendizaje de la humanidad, Siep distingue diversos grados de reconocimiento:

1. Respetar la integridad (física y psíquica) del otro.

2. No discriminar al otro, no separarlo o rebajarlo.

3. Tolerancia: Aceptar en el propio entorno a uno que de entrada no pertenece a él, preservando su otredad, su extrañeza.

4. Solidaridad: Pasando a una tolerancia positiva, apoyar, ayudar al otro a que pueda satisfacer sus necesidades, desplegar sus capacidades y posibilidades.

5. Amistad: cuando el apoyo va ligado a una estima y a un afecto recíprocos.

6. Promoción de un 'cosmos' social y natural, ecología social y natural: Interés en la otredad enriquecedora del otro, y también la vinculación de horizontes diversos en una obra común (Siep 2009, 24-26).

Obviamente estos grados no están todos al mismo nivel. Así los tres primeros son deberes y por tanto no pueden ponerse a la ponderación de otros individuos o grupos. También los demás tienen derecho a cierta medida de solidaridad. Una mayor medida de apoyo recíproco, de amistad y de cooperación en tareas comunes pueden considerarse como «deberes meritorios» o supererogatorios, a los que uno no puede ser obligado jurídicamente (Siep 2009, 26).

\section{III.3. Patrones de Reconocimiento}

Más cercano al texto hegeliano, pero haciendo una construcción propia, A. Honneth propone una gradación de formas de reconocimiento, que es a la vez una sistemática. El proceso se inicia con la autoexperiencia instrumental: el sujeto tiene que satisfacer sus necesidades y para ello entrar en el trabajo. Éste representa el primer paso en la formación del sujeto, que conlleva una cierta cosificación de sí mismo. Es un primer paso para el reconocimiento, que debe ser ampliado para superar la cosificación; para ello se requiere un reconocimiento recíproco. El primer reconocimiento es el amor entre hombre y mujer, esposo y esposa (Honneth 1994, 60-62). Para caracterizar una tal relación del conocerse recíproco en el otro, Hegel aplica por primera vez el concepto de reconocimiento. Pero el amor tiene a nivel subjetivo sus contraindicaciones, por lo que es substituido por un «racional sentimiento de solidaridad». La relación de amor se amplía con otras dos relaciones: la posesión familiar y el nacimiento de la descendencia (Honneth 1994, 63-68). 
Toda convivencia humana presupone una cierta afirmación recíproca elemental entre los sujetos, que puede significar una cierta limitación. Esta es la primera figura implícita de conciencia jurídica. El reconocimiento jurídico se empieza a asegurar por medio de un contrato social, el cual puede ser superado mediante la adhesión solidaria (Honneth 1994, 73, 151). A partir de la diferenciación de estos tres grados o patrones de reconocimiento recíproco: 1) dedicación emocional, relaciones de amor y amistad; 2) reconocimiento jurídico; 3) adhesión solidaria, se distinguen tres ámbitos en los que se desarrollan, que son familia, sociedad civil y Estado. Honneth estima que lo específico de Hegel (y de Mead) consiste en reconducir las tres esferas de interacción a patrones diferentes del reconocimiento recíproco: amor, derecho y solidaridad (Honneth 1994, 151 s. / 116 s.).

En este caso el amor no necesariamente ha de entenderse en el sentido de relación íntima sexual, según su revaluación romántica, sino todas las relaciones primarias, mientras se puedan entender según el patrón de las relaciones eróticas entre dos, amistades y relaciones paterno-filiales, consistentes en fuertes lazos afectivos entre pocas personas. En este sentido es que el amor es el primer grado del reconocimiento recíproco, el primer reconocimiento del propio sí mismo en el extraño (Sein Selbstsein in einem Fremden). ${ }^{5}$

«Por solidaridad, en una primera anticipación, puede entenderse un tipo de relación de interacción en el que los sujetos recíprocamente participan en sus vidas diferenciables, porque se valoran entre sí en forma simétrica» (Honneth 1994, 208 / 157). Esta descripción muestra que se aplicará la solidaridad especialmente en las relaciones de grupo, que surge de la experiencia de una resistencia común contra la opresión política. La vinculación al grupo ha cambiado gracias el movimiento de mayor individualización, donde pesa más la autoestima, la autoconfianza, el sentimiento del propio valor. Ello no impide que en una sociedad posttradicional se pueda hablar de solidaridad social. «En las condiciones de la sociedad moderna, la solidaridad está por ello ligada al presupuesto de relaciones sociales de valoración simétrica entre sujetos individualizados (y autónomos); en este sentido, valorarse simétricamente significa considerarse recíprocamente a la luz de los valores que hacen aparecer las capacidades y cualidades de cualquier otro como significativas para la praxis común.» (Honneth 1994, 209 / 158).

[5] Honneth 1994, 153-172. Sobre la relación jurídica, pp. 173-195. 


\section{Conclusión}

Quisiera cerrar estas consideraciones con una cita de la Filosofía Real, donde a mi parecer se puede ver en resumen qué se entiende por reconocimiento y la cercanía de este concepto al de solidaridad. Escribe Hegel (GW VIII, 215 / 176):

«En el acto de reconocer el sí mismo deja de ser esta singularidad; en el reconocimiento el sí mismo alcanza dimensión jurídica, es decir: que su existencia ha dejado de ser inmediata. Lo reconocido es reconocido como valiendo inmediatamente, por su mero ser; pero precisamente este ser está producido a partir del concepto, es ser reconocido; el hombre es reconocido necesariamente y necesariamente reconoce. Esta necesidad es suya propia, no pertenece a nuestro pensamiento por oposición al contenido. En el acto de reconocer el hombre mismo es el movimiento y este movimiento supera precisamente su estado de naturaleza, es reconocer, lo natural sólo es, no es espiritual».

Intentemos desgranar algunas afirmaciones:

1. En el acto de reconocer uno va más allá de su propia singularidad, «deja de ser esta singularidad». No por ello pierde su autonomía, pero sí su aislamiento y pasa a entenderse y a obrar como autónomo/libre en una comunidad de autónomos/libres. Lo cual no sólo no significa pérdida de su autonomía, sino su reconocimiento por otros, por la comunidad en la que uno se encuentra inserido.

2. La primera figura «objetiva», pública, de carácter social o político -ello no implica que antes o simultáneamente se dé la del amor- es el reconocimiento jurídico. Es la relación mínima con los que no se tienen relaciones, lo que Siep ha llamado «solidaridad básica» (Grundsolidarität) (Siep 2009, 23 s.).

3. El reconocimiento es inmediato, no requiere avales ni presentación de méritos, «lo reconocido es reconocido como valiendo inmediatamente, por su mero ser», por ser quien es, a saber, individuo humano. La misma inmediatez caracterizará la persona (FD \$ 34$)$.

4. El reconocimiento no es algo puramente natural, instintivo, sino algo fruto de la libertad, algo ético. «Precisamente este ser está producido a partir del concepto, es ser reconocido»; es algo puesto no dado puramente por la naturaleza. El reconocimiento nos sitúa claramente en un ámbito ético.

5. La relación es recíproca: «el hombre es reconocido necesariamente y necesariamente reconoce». Se trata de la reciprocidad esencial en el reconocimiento, que no siempre se da en la solidaridad, o por lo menos no en todas sus formas. Aunque ni siquiera en los casos que más claramente destaca el carácter gratuito del reconocer y de la solidaridad no puede 
descartarse alguna relación recíproca, ya que como humanos siempre estamos en deuda con otros. El carácter necesario del reconocimiento y de la solidaridad no hace más que aludir a su carácter antropológico, esencial, del cual deriva su carácter ético. Uno es solamente en la medida en que es con otros, co-es; incluso el sentimiento de la más extrema soledad es una forma de experimentar mi ser con los demás.

El reconocimiento consigue inserir el otro en el mismo sí mismo de uno mismo. Este es su mayor logro, con lo cual los otros, la sociedad, no son algo extraño a uno mismo, sino que configuran la propia identidad. A la vez esta conexión con los otros deriva del propio sí mismo, de modo que para la misma afirmación de mi autonomía y libertad se requiere del otro, de la comunidad. Necesito de los demás para la misma afirmación y ejercicio de la propia autonomía y libertad.

\section{BiBLIOGRAFÍA}

Amengual, Gabriel (1990), "Anerkennung", en Europäische Enzyklopädie zu Philosophie und Wissenschaften, hrsg. v. H.J. Sandkühler, 4 vols. Hamburgo: Meiner, 1990, vol. I, pp. 128-131.

Amengual, Gabriel (2000), «Individualismo y comunitarismo en la Filosofía del Derecho de Hegel», in: Mariano Álvarez Gómez / M. del Carmen Paredes Martín (eds.), Razón, libertad y Estado en Hegel. I Congreso Internacional de la Sociedad Española de Estudios sobre Hegel. Salamanca, 5-9 mayo 1998. Salamanca: Ed. Universidad, 2000, pp. 49-58.

Aristóteles, Etica nicomáquea, en Etica nicomáquea. Etica eudemia. Traducción de J. Palli Bonet. Madrid: Gredos 1985.

Boder, Remo (1987), Scomposizioni. Forme dell'individuo moderno. Torino: Einaudi, 1987

D’Abbiero, Marcella / Vinci, Paolo (ed.) (1995), Individuo e modernitá. Saggi sulla filosofia di Hegel. Milano: Guerini 1995.

DüsING, Edith (1986), Intersubjektivität und Selbstbewusstsein. Behavioristische, phänomenologische und idealistische Begründungstheorien bei Mead, Schütz, Fichte und Hegel. Köln: Dinter, 1986.

GW VIII: HegeL, Jenaer Systementwürfe III. Unter Mitarbeit von Johann Heinrich Trede hrsg. v. Rolf-Peter Horstmann (Gesammelte Werke, vol. VIII), Hamburgo: Meiner, 1976; vers. cast.: Filosofía Real. Edición de José María Ripalda. Madrid: FCE, 1984. Esta obra se citará en el texto, indicando el volumen VIII de las GW y el número de página y separada con una / el número de la página de la traducción castellana.

FDE: HeGEL, Phänomenologie des Geistes, hrsg. v. Hans-Friedrich Wessels und Heinrich Clairmont. Hamburgo: Meiner, 1988 (Philosophische Bibliothek, 414); vers. cast.: Fenomenología del espíritu. Trad. de W. Roces con la 
colaboración de R. Guerra. México: FCE, 1971. Esta obra se cita con FDE y el número de página de la edición alemana y el de la castellana, separado con una $/$.

FD: Hegel, Grundlinien der Philosophie des Rechts: Werke, hrsg. v. E. Moldenhauer y K.M.Michel, vol. VII. Frankfurt/M: Suhrkamp, 1970.

Honneth, Axel (1994), Kampf um Anerkennung. Zur moralischen Grammatik sozialer Konflikte. Fráncfort: Suhrkamp, 1994. La lucha por el reconocimiento: por una gramática moral de los conflictos sociales. Barcelona: Crítica, 1997.

Jonas, Hans, El principio de la responsabilidad. Barcelona: Herder 1994.

MazA, Luís Mariano de la (2009), «El sentido del reconocimiento en Hegel», Revista latinoamericana de filosofía 35 (2009: 2) edición on-line.

SieP, Ludwig (1974), “Der Kampf um Anerkennung. Zu Hegels Auseinandersetzung mit Hobbes in der Jenaer Schriften", Hegel-Studien 9 (1974) 155-207.

SieP, Ludwig (1979), Anerkennung als Prinzip der praktischen Philosophie. Untersuchungen zu Hegels Jenaer Philosophie des Geistes. Freiburg/ München: Alber 1979 [nueva edición: Hamburgo: Meiner 2014].

SIEP, Ludwig, (2000), Der Weg der Phänomenologie des Geistes. Ein einführender Kommentar zu Hegels „Differenzschrift" und „Phänomenologie des Geistes“. Darmstadt: WBG, 2000; vers. castellana: El camino de la fenomenología del Espíritu. Un comentario introductorio al «Escrito sobre la Diferencia» y la «Fenomenología del Espíritu» de Hegel. Barcelona: Anthropos 2015.

SIEP, Ludwig (2009), „Anerkennung zwischen Individuen und Kulturen“, en Annemarie Gethmann-Siefert, ElisabethWeisser-Lohmann (Hrsg.), Wege zur Wahrheit. Otto Pöggeler zum 80. Geburtstag. München: Fink 2009, pp. 15-31.

Valls Plana, Ramon (1971), Del yo al nosotros. Lectura de la 'Fenomenología del espíritu’ de Hegel. Barcelona: Laia, 1971 (Barcelona: PPU 1996). 